\title{
Automating indicator data reporting from health facility EMR to a national aggregate data system in Kenya: An Interoperability field-test using OpenMRS and DHIS2
}

\author{
James M. Kariuki ${ }^{1}$, Eric-Jan Manders ${ }^{1}$, Janise Richards ${ }^{1}$, Tom Oluoch ${ }^{2}$, Davies Kimanga ${ }^{3}$, Steve \\ Wanyee ${ }^{4}$, James O. Kwach ${ }^{2}$, Xenophon Santas ${ }^{1}$
}

1. Division of Global HIV \& TB, Center for Global Health, Centers for Disease Control and Prevention, Atlanta, USA

2. Centers for Disease Control and Prevention-Kenya, Nairobi, Kenya

3. National AIDS \& STI Control Programme (NASCOP), Nairobi, Kenya

4. The International Training and Education Center for Health (I-TECH) - Kenya, Nairobi, Kenya

\begin{abstract}
Introduction: Developing countries are increasingly strengthening national health information systems (HIS) for evidence-based decision-making. However, the inability to report indicator data automatically from electronic medical record systems (EMR) hinders this process. Data are often printed and manually reentered into aggregate reporting systems. This affects data completeness, accuracy, reporting timeliness, and burdens staff who support routine indicator reporting from patient-level data.
\end{abstract}

Method: After conducting a feasibility test to exchange indicator data from Open Medical Records System (OpenMRS) to District Health Information System version 2 (DHIS2), we conducted a field test at a health facility in Kenya. We configured a field-test DHIS2 instance, similar to the Kenya Ministry of Health (MOH) DHIS2, to receive HIV care and treatment indicator data and the KenyaEMR, a customized version of OpenMRS, to generate and transmit the data from a health facility. After training facility staff how to send data using DHIS2 reporting module, we compared completeness, accuracy and timeliness of automated indicator reporting with facility monthly reports manually entered into $\mathrm{MOH}$ DHIS2.

Results: All 45 data values in the automated reporting process were $100 \%$ complete and accurate while in manual entry process, data completeness ranged from $66.7 \%$ to $100 \%$ and accuracy ranged from $33.3 \%$ to 95.6\% for seven months (July 2013-January 2014). Manual tally and entry process required at least one person to perform each of the five reporting activities, generating data from EMR and manual entry required at least one person to perform each of the three reporting activities, while automated reporting process had one activity performed by one person. Manual tally and entry observed in October 2013 took 375 minutes. Average time to generate data and manually enter into DHIS2 was over half an hour ( $M=32.35$ mins, $S D=0.29)$ compared to less than a minute for automated submission $(M=0.19$ mins, $S D=0.15)$.

Discussion and Conclusion: The results indicate that indicator data sent electronically from OpenMRS-based EMR at a health facility to DHIS2 improves data completeness, eliminates transcription errors and delays in reporting, and reduces the reporting burden on human resources. This increases availability of quality indicator data using available resources to facilitate monitoring service delivery and measuring progress towards set goals. 
Keywords: Health information systems, electronic medical records, interoperability, indicator reporting, DHIS2, OpenMRS, health information exchange, data aggregation

Correspondence: Email- jkariuki@cdc.gov, Phone- + (1)-(404) 718-8349, Address- 1600 Clifton Road, MS-E77 Atlanta, GA 30329, USA

DOI: 10.5210/ojphi.v8i2.6722

Copyright (C2016 the author(s)

This is an Open Access article. Authors own copyright of their articles appearing in the Online Journal of Public Health Informatics. Readers may copy articles without permission of the copyright owner(s), as long as the author and OJPHI are acknowledged in the copy and the copy is used for educational, not-for-profit purposes.

The findings and conclusions in this report are those of the authors and do not necessarily reflect the views of the US Centers for Disease Control and Prevention or the Kenyan Government.

\section{Introduction}

The fight against HIV has played a major role in the implementation and use of Health Information Systems (HIS) in many low and middle-income countries for management of longitudinal health records. The number of HIV patients enrolled on antiretroviral therapy (ART) has increased exponentially over the last ten years due to improved access to HIV testing and revised guidelines requiring early initiation of ART among those infected [1-5]. This has accelerated further adoption and scale up of electronic medical records (EMR) and monitoring and evaluation (M\&E) information systems to monitor patient and program outcomes [6]. International donor organizations, such as the Joint United Nations Programme on HIV/AIDS' (UNAIDS) and the United States Government's President's Emergency Plan for AIDS Relief (PEPFAR), have set ambitious goals towards ending the epidemic and developed comprehensive indicators for monitoring progress made through various programs [7-9]. Achieving these goals requires a shift to a data-driven approach that uses data from the national level down to the service delivery (health facility) level.

eHealth is a key enabler and driver of improved health outcomes and an essential infrastructure to support information exchange between all participants in the health care system [10,11]. The ability to use information for monitoring health service delivery, planning programs, reporting health indicators, measuring achievement, and improving accountability requires timely, reliable, high-quality, and accessible health service data. These are key to realizing global health goals, especially in resourcelimited settings [12-14]. Effectively managing health data requires robust HIS, including both an EMR to manage patient records and an aggregate data system for M\&E. Although some countries have adopted and implemented both EMR and aggregate system with national coverage, they tend to be standalone or silo systems [15]. Interoperability among HIS is essential to achieving health goals by facilitating the availability and use of quality health data.

Despite increased adoption of electronic HIS, the lack of data exchange remains a challenge to data quality and availability [16]. Printing electronic data from one system and re-entering it into another system manually is commonplace. Manual data entry is labor intensive and prone to transcription errors. It increases the time from when the indicator data are generated in the EMR to its availability in the aggregate data system, and increases the workload for health workers responsible for reporting $[17,18]$. As data demand increases, limited-resource sites may struggle to hire and sustain the staff 
needed to support manual data reporting $[14,19]$. All these factors can potentially affect the ongoing monitoring of health programs, planning and resource allocation for health services, and delivery of quality and efficient healthcare services.

In earlier work, we conducted a laboratory-based study examining the feasibility of automating reporting of a subset of PEPFAR's next generation indicators from Open Medical Records System (OpenMRS), an open-source medical records system [20], to District Health Information System version 2 (DHIS2), a tool for collection, validation, analysis, and presentation of aggregate statistical data, tailored to integrated health information management activities [21]. This study demonstrated that data generated from OpenMRS and sent electronically to DHIS2 can maintain the accuracy and completeness needed to develop appropriate indicators [22]. It also indicated that an automated indicator reporting process had the potential to provide timely health information and reduce staff workload. In this work, we extend our findings to conduct an experiment with a field-based study exploring the impact of adopting HIS interoperability at the facility level.

\section{Methods}

\section{Field-test study design}

We designed a mixed-method field test to compare human resources reporting efforts, data accuracy and completeness, and timeliness of submitting data reports from the health facility to the national health management information system (HMIS) for manual and automated indicator data reporting processes.

We conducted the study in two phases and used four data collection methods. In the first phase, we developed, tested, and implemented the automated software, while in the second phase we examined the automation's impact. The four data collection methods were:

1. Document review of Kenya MOH HIV facility reporting tools and National AIDS \& STI Control Programme (NASCOP) indicator manual to identify indicators to automate and understand how they are calculated;

2. Desk review of monthly reports at the facility and national levels to audit data quality and compare reporting time between the two phases for manual and automated reporting;

3. Focus group discussion with health facility staff to gain their perspectives on the data collection, aggregation and submission process; and

4. Observation of the manual reporting process to determine the data collection and aggregation procedures used by the staff.

\section{Definitions of variables measured}

For this study, we defined three variables:

1. Human resources are the staff required to support indicator data reporting from the facility EMR to the national HMIS.

2. Data quality focuses on data accuracy and completeness characteristics of indicator data sent from the facility to the national HMIS for manual or automated reporting. This is 
calculated as a percentage of complete data values entered into national HMIS accurately out of the expected data values [23].

- Data completeness is the degree to which values of all selected indicator data elements in the facility monthly reports, generated from EMR or manual tally, are available in the national HMIS.

- Data accuracy is the degree of concordance between indicator data values in the facility monthly reports, generated from EMR or manual tally, with data values in the national HMIS.

3. Reporting time is the time taken to prepare and submit indicator data reports into the national HMIS from the facility.

\section{Study Setting}

We identified Kenya for the field-test study because DHIS2, a tool for collection, validation, analysis, and presentation of aggregate statistical data, and OpenMRS, an open-source medical records system, are currently in use and supported by $\mathrm{MOH}$. Within Kenya, we selected Kisumu East District Hospital comprehensive care clinic (CCC) because it met our three inclusion criteria: 1) large number of electronic patient records (over 3,000 patients enrolled on HIV treatment), 2) Kenya MOH support, and 3) established EMR and data entry processes. Kisumu East District Hospital CCC is relatively large (approximately 13,185 adult and pediatric patients were receiving HIV care and treatment at the time of the study) and is operated by the Kenyan MOH. The hospital has used KenyaEMR (a customized version of OpenMRS) for more than one year as a point-of-care system and for retrospective data entry of routine patient data.

\section{Field-test study data collection}

We collected data on manual and automated reporting work processes following the steps shown in Figure 1.

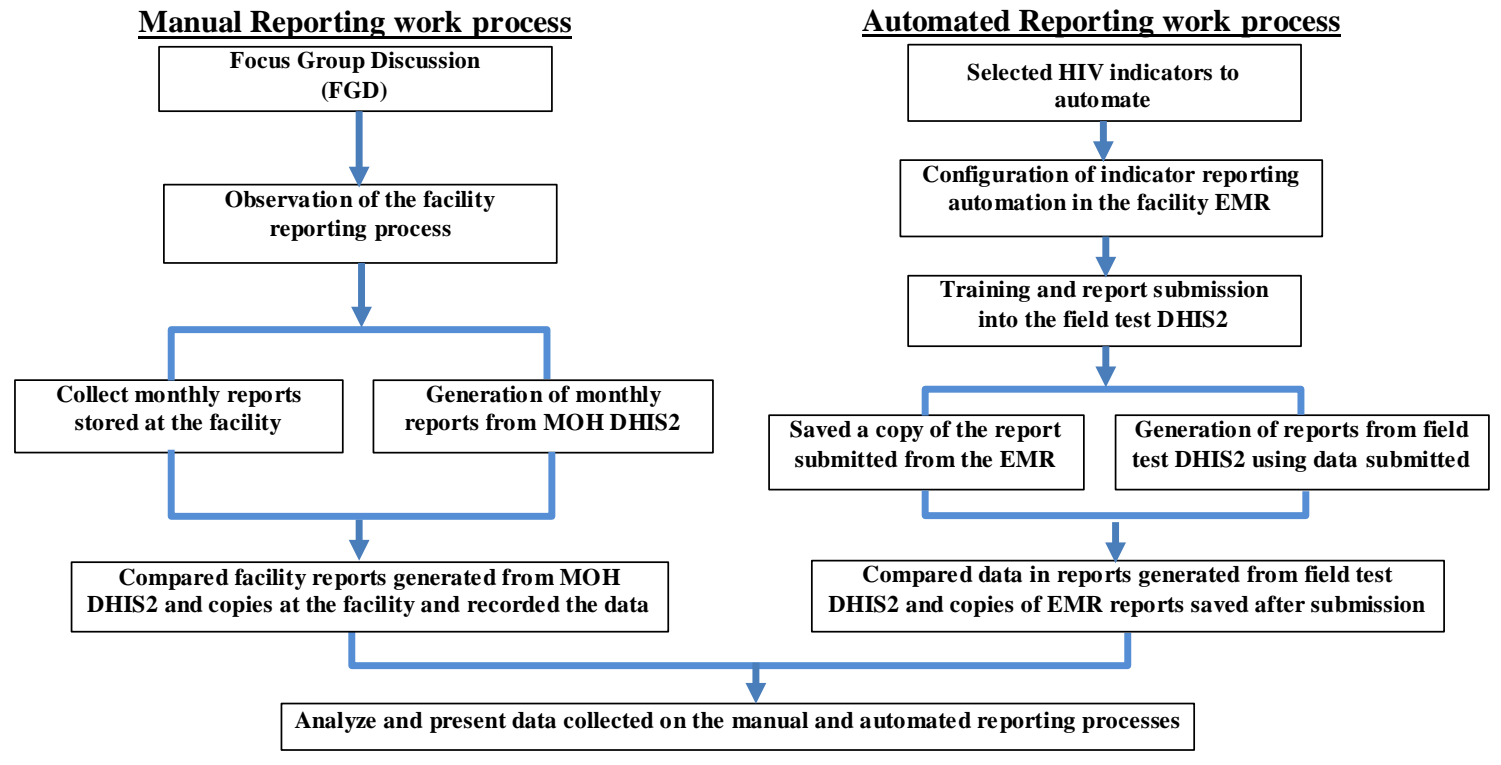

Figure 1: Indicator data reporting field-test study work process 


\section{Manual reporting work process}

Focus group discussion (FGD): We conducted a FGD with seven key staff who use KenyaEMR routinely and are responsible for HIV indicator reporting at the facility. These staff included clinical officers, nursing officers, health records and information officer (HRIO), and data clerks at the HIV clinic. During the FGD, we collected information about EMR use, current indicator data reporting process to MOH DHIS2, and human resources effort required for reporting (i.e., the number of staff and duration per month).

Observation of the facility reporting process: We observed the current reporting process by shadowing the facility's data clerk and HRIO while they prepared the routine monthly HIV care and treatment report. We documented the process and recorded the approximate time it took to accomplish each step using a stopwatch. In addition, we produced the monthly indicator data report in KenyaEMR and recorded the time to generate a report during the study period.

Manual reporting data quality desk review: We collected copies of the HIV care and treatment indicator reports from July 2013 to January 2014 at the facility (see Appendix 1 for a sample reporting form) and produced the facility's monthly indicator reports from MOH DHIS2 for the same timeframe. Then, we transferred the data in the monthly reports into the data quality comparison tool, (Figure 2) to compare the completeness and accuracy of indicator data submitted to MOH DHIS2 and the reports at the facility. We counted the number of data elements with accurate values and data elements with transcription errors or missing values in the two reports and summarized the results in the same tool. For manual reporting, we used the report at the facility as reference.

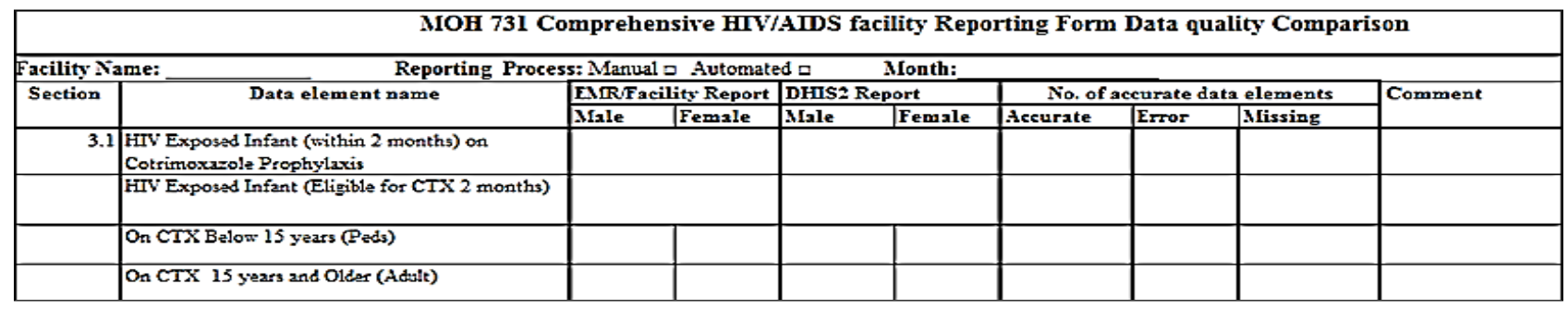

Figure 2: Data quality comparison tool

\section{Automation implementation}

Selection of HIV indicators to automate: First, we reviewed the MOH Comprehensive HIV/AIDS Facility Reporting Form (Appendix 1) to identify HIV care and treatment indicators to automate [24]. Selected indicators had data routinely collected in KenyaEMR at the facility, including all data elements required to generate aggregate data values.

Indicator data automation configuration in the facility KenyaEMR: To ensure that the study did not interrupt the normal facility monthly reporting to $\mathrm{MOH}$, we created a separate DHIS2 instance with identical data elements in the MOH DHIS2 using the OpenMRS to DHIS2 indicator automation guide developed in the previous study [25]. Next, we generated an XML report definition template with the HIV care and treatment data elements. Then we mapped the data elements in the report definition template to the PEPFAR HIV care and treatment indicators to enable us reuse the SQL code created during the feasibility study $[22,26]$. Using an SQL editor, we created query statements to generate each 
data element value from KenyaEMR and add them to the XML report definition template. Next, we loaded the DHIS2 reporting module into the facility KenyaEMR and uploaded the XML report definition template embedded with SQL queries. Finally, we connected the two systems by adding field-test DHIS2 login and link details to the KenyaEMR.

Training and report submission into the field-test DHIS2: To ensure a successful implementation, we trained facility staff responsible for reporting on sending indicator data electronically from KenyaEMR to field-test DHIS2 using the DHIS2 Reporting Module. The facility staff generated and transmitted HIV care and treatment indicator data for seven months, July 2013 to January 2014, from the facility KenyaEMR to the field-test DHIS2. After transmission, a report containing indicator data values and transmission results for each month was saved on a computer. We also recorded the time to generate and transmit each report from the KenyaEMR to the field-test DHIS2.

Automated reporting data quality desk review: To verify the completeness and accuracy of the automated indicators, we produced HIV care and treatment indicators data reports from the field-test DHIS2 and printed the reports submitted automatically from the KenyaEMR for the seven months. Then, we compared the data in the two reports for each month. We counted the number of indicator data elements with identical values, transcription errors or missing in the two reports and recorded the results in the data quality comparison tool (Figure2, above). In the automated reporting process, we used the report produced in KenyaEMR at the facility as reference.

\section{Analysis}

We developed a workflow diagram of the current reporting process at the facility using the data captured during the FGD and observation notes. We reviewed the workflow with facility staff to confirm that it captured the current reporting process (see Current Reporting Process in Figure 3).

For the descriptive data analysis, we reviewed data in the data quality comparison tool and summarized the data on data quality dimensions. Then we calculated the percentage of complete and accurate indicator data values entered into the national HMIS out of the total indicator data values expected each month to compare data quality between the manual and automated reporting processes. We also reviewed the observation notes and tabulated data on time to produce and submit reports for each month during the study period in Microsoft Excel. Then we calculated the average time required to generate and submit the report for each reporting process. Finally, we developed workflow diagrams on human resources, graphs to visualize data completeness and accuracy, and a timeline graphic with mean, median and interquartile range to visualize data reporting time for manual and automated reporting processes.

\section{Results}

\section{Facility reporting process}

Data were entered into the facility EMR by clinicians during patient visit or retrospectively by data clerks. These data were also captured in paper registers. When planning the field-test, we expected that facility staff generated and printed indicator data reports from patient data in the KenyaEMR, then manually entered it into MOH DHIS2. However, the FGD and observations indicated that staff manually tallied HIV indicator data from several registers to compile the $\mathrm{MOH}$ reporting form before 
manual entry into MOH DHIS2, bypassing the KenyaEMR entirely (see Current Reporting Process in Figure 3).

In terms of staffing responsible for HIV reporting, one receptionist/data clerk prepares HIV indicator reports every month, and one of the three health records and information officers (HRIOs) at the facility reviews and submits it to MOH DHIS2. The HRIOs have access to MOH DHIS2 through the facility's internet and can manually enter and submit facility’s indicator data reports to $\mathrm{MOH}$.

\section{Human resources burden comparison for manual and automated reporting}

This study compares the human resources needed for manual and automated indicator reporting activities. The current reporting process has five activities, each requiring at least one person to complete. The data clerk collects registers from various clinics, tallies and aggregate value for each indicator, compiles the monthly indicator report form, and sends it to the HRIO office. The facility HRIO reviews the report, then manually enters the data into DHIS2 (see Current Reporting Process in Figure 3).

The expected reporting process has three activities, each requiring at least one person to complete. The data clerk generates the monthly indicator data report from KenyaEMR, prints it, and sends it to HRIO office. The facility HRIO reviews the report, then manually enters the data into DHIS2 (see Expected Reporting Process in Figure 3).

The automated reporting process has one activity to generate, review, and submit the indicator report electronically into DHIS2. This activity can be completed by one HRIO within the EMR (see Automated Reporting Process in Figure 3).

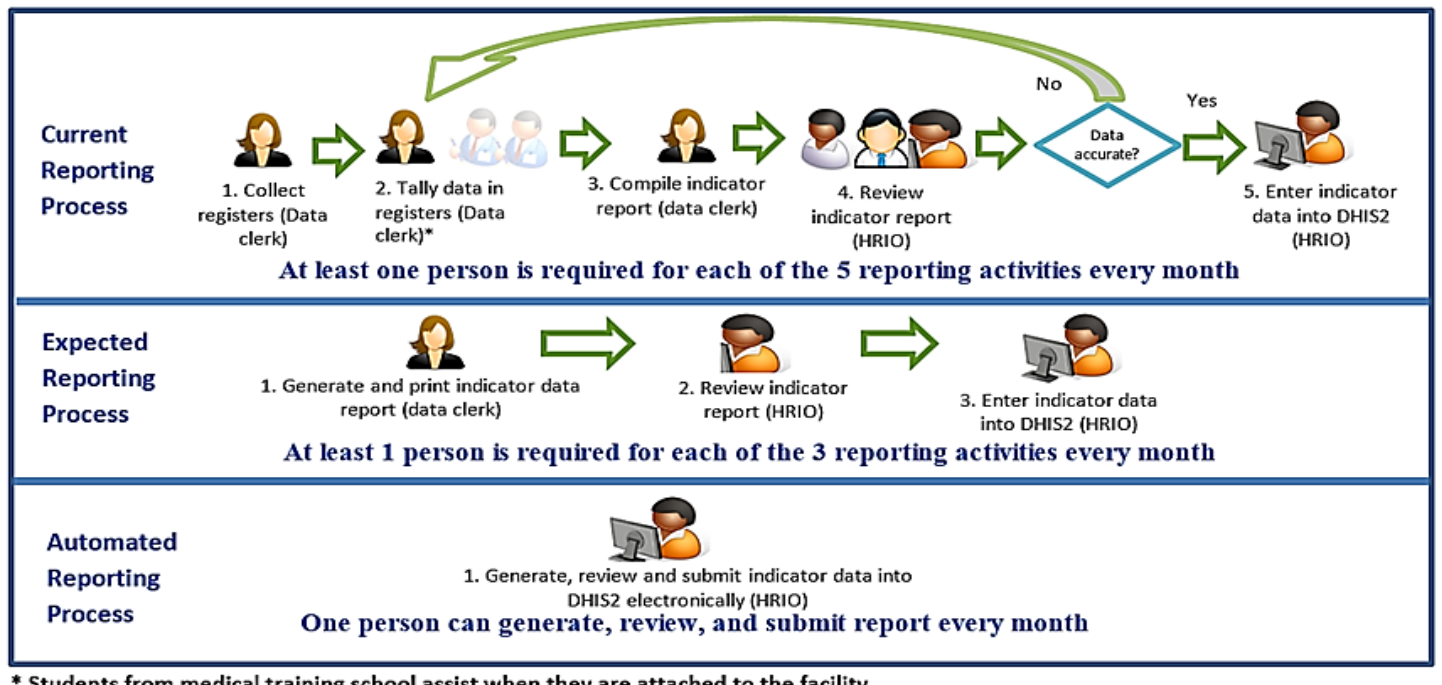

Figure 3: Comparison of human resources required for the three reporting processes 


\section{Comparison of data completeness and accuracy between manual and automated reporting processes}

Using the selection criteria, we identified eight indicators for automation in the HIV care and treatment section of the $\mathrm{MOH} 731$ reporting form. Including disaggregates; these eight indicators had 45 data elements. Data values for all 45 selected indicator data element submitted electronically from KenyaEMR to DHIS2 for the seven months were 100\% complete and accurate. Manually entered indicator data averaged 89\% completeness (ranging from 66.7\% to 100\%) and 71\% accuracy (ranging from $33.3 \%$ to $95.6 \%$ ). This indicates that during manual data entry, some indicator data values were not entered and transcription errors were introduced. Figure 4 shows the completeness and accuracy for both processes during the seven-month study period.

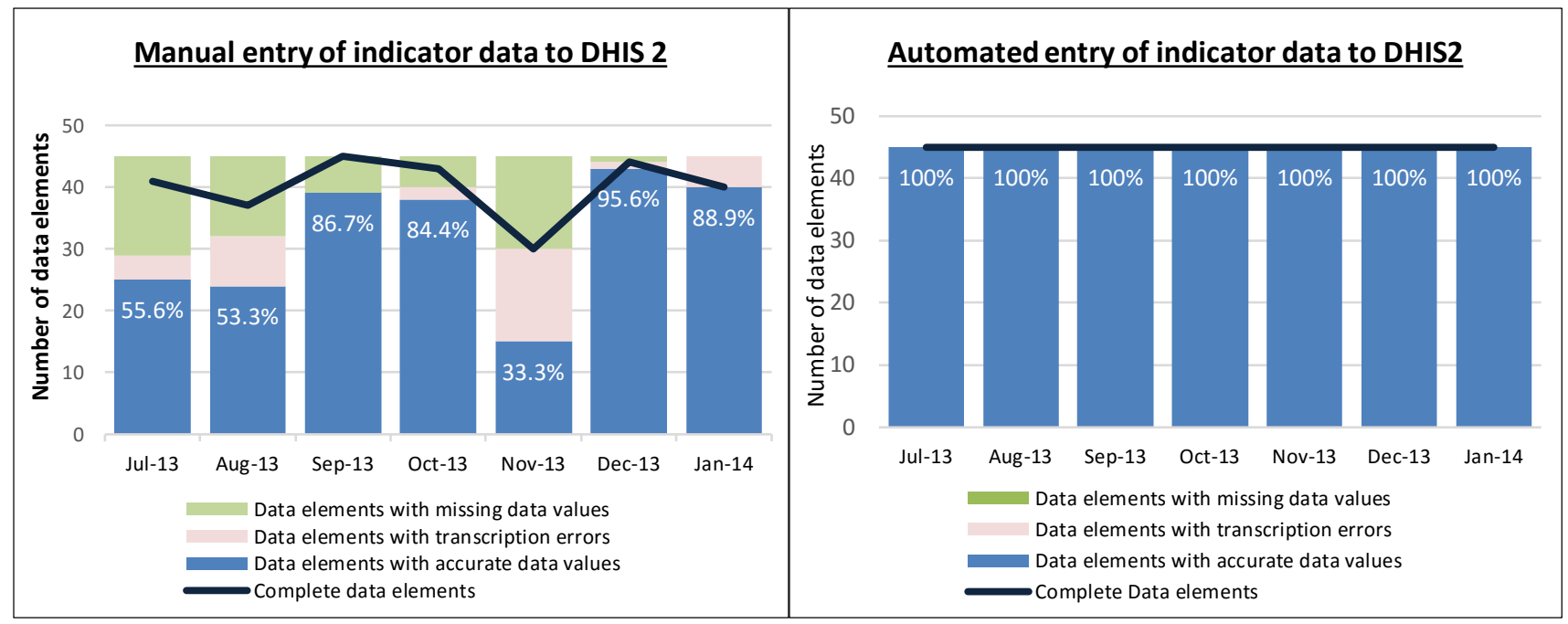

Figure 4: Comparison of completeness and accuracy between manual and automated data elements

\section{Comparison of indicator data reporting time between manual and automated reporting}

Figure 5 shows the time required to prepare and get indicator data into DHIS2 for each reporting process. While this study assumes of data contained in KenyaEMR, it includes time for the current manual reporting process observed in October 2013. Of the 375 minutes needed for the current process, approximately 345 minutes (92\%) of the total time was used to aggregate and compile indicator data. The 30 minutes required to enter indicator data values manually into DHIS2 were comparable to the expected reported process. 


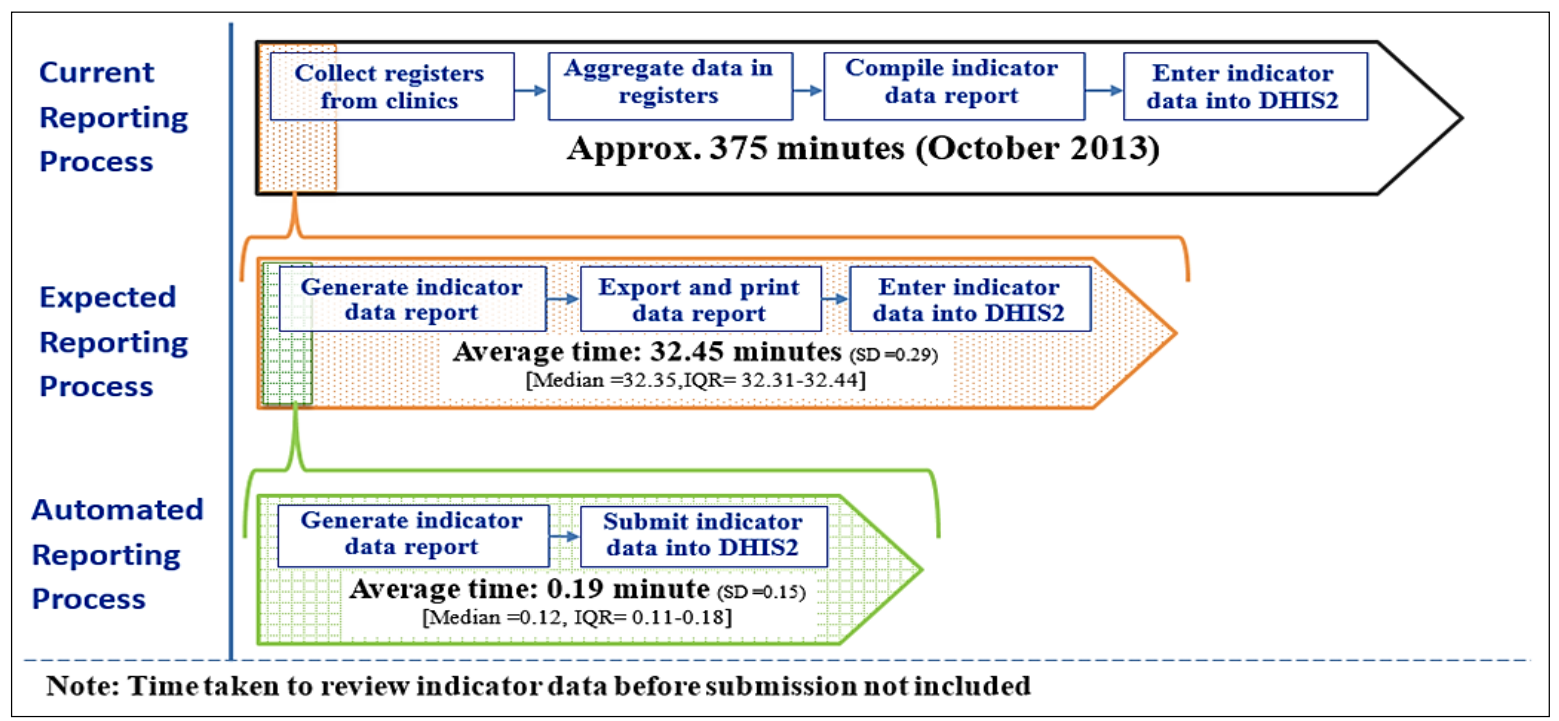

Figure 5: Timeline comparing time to generate and submit indicator data into DHIS2 using manual and automated processes

\section{Discussion}

The study findings indicate that it is feasible and beneficial to automate indicator data reporting from EMRs to aggregate data systems, and that implementing automated process improves the completeness and accuracy of indicator data reports. In addition, the results validate that automating reporting reduces the facility's human resource burden by eliminating manual data entry, which is time and labor intensive and prone to human error [18].

The automated reporting process tasks required one staff member to complete one task, while the manual reporting process required at least one staff for each of the three activities. Often staff are pulled away from their health care-related responsibilities to prepare reports. As data demand increases, highvolume sites may not be able to sustain the human resources needed to support manual data reporting. With automated reporting, existing staff can generate and send reports from the EMR to the national HMIS, eliminating the need for additional staff. Surprisingly, this study discovered that facility staff were bypassing KenyaEMR and spending more than half a workday (5.75 hours) aggregating reporting data from paper records by hand each month. While out of scope for this study, this indicates a need for further investigation.

Automated entry improved timely availability and quality of indicator data, consistent with studies on automated entry of surveillance data $[17,18]$. While aggregate data was complete using the automated process, data completeness in the manual system was only about two-thirds. Not only was the data accuracy much higher for automated data entry than for manual data entry, there was also a substantial difference in the time required to generate and submit data between the manual and automated reporting processes. This is fundamental to both monitoring progress toward meeting performance targets, planning, and resource allocation, and identifying areas needing additional support to improve health outcomes and impact. 
While there is a great promise with indicator reporting automation, a number of issues need to be addressed to ensure successful implementation. During the transition from paper-based systems, some patients' data or records may not be in the EMR $[27,28]$. This requires proper planning to ensure that key data for automated reporting is in the EMR or the ability to coordinate reporting using different methods. Procedures for routine data quality assurance and audits in the EMR are necessary to ensure data exchanged is of acceptable quality. Staff will require training and active engagement to adjust to new work practices and workflows. Robust information technology infrastructure (including reliable electric power, adequate computers and Internet access) and support at the facility are critical to ensure consistent EMR availability. In addition, staff should actively identify and share lessons learned and best practices with other facilities, $\mathrm{MOH}$, and funders to help improve use of the EMR and system interoperability [29].

Furthermore, there is a need to assess feasibility of automating indicator data reporting from other EMRs used in resource-limited settings to national HMIS. This will consolidate information to guide development of standardized indicator data reporting, as well as identify the best approaches to support scale-up of electronic health information systems with available resources.

Limitations: Our study had a few limitations. We were not able to observe and record the time taken to review reports before data entry into DHIS2, which would have provided additional information when comparing the manual and automated reporting processes. Therefore, we excluded the time to taken to review indicator data reports on all processes to ensure consistency. While quality of data in the EMR is important, in this study we defined data quality as the completeness and accuracy of aggregate data transmitted from KenyaEMR to DHIS2 (e.g., the data sent was the same as the data received.) The quality of the patient-level data used to calculate the indicator was not assessed. We assumed that the study site experienced similar data quality challenges reported in other studies conducted in resource-limited settings [16,30,31]. In addition, only one facility was used in this study, which limits our ability to generalize the results widely. This effect was minimized by selecting a facility that is typical of HIV care and treatment facilities in Kenya and uses the same indicator reporting process. The large number of patients enrolled at our study site enabled us make observations that would be expected in other busy, yet understaffed health facilities.

\section{Conclusion}

This study demonstrates that sending indicator data automatically from a health facility EMR (based on OpenMRS) to the national-level reporting system (DHIS2) is both possible beneficial. It eliminates need for manual data entry that can introduce transcription errors and reduces delays, thus improving indicator data completeness and accuracy for use at the facility, subnational, and national levels. It also reduces the amount of time to prepare and submit indicator data and the number of facility staff required to fulfil reporting requirements at health facilities, which is key to scale up of HIS without the need for additional human resources.

There is potential to increase indicator data completeness, accuracy and availability in the national HMIS. Additionally, increasing the focus on automated indicator data reporting may facilitate the development of internationally recognized data exchange standards for aggregate data, which is fundamental to monitoring global health outcomes and impact. Further studies should be conducted on the effect of the use of data exchange standards for automated reporting using different EMRs on data quality and timeliness. 


\section{Acknowledgements}

Thanks to Kisumu East District Hospital for allowing us to conduct the field-test study. To Molly Oloo, Rinnie Juma, and Moureen Ogonda, thank you for providing information on reporting and your support at the facility during the study. We would like to thank and George Owiso, John Gitahi, Nicholas Ingosi, Benard Otieno, and Prisca Teka from I-TECH Kenya for their technical and logistics support; Bob Jolliffe and Thái Chương, working with HISP-India, for sharing information on DHIS2 reporting module code and insights on how to implement it; and the CDC public health informatics research laboratory, which provided infrastructure. Thanks to Jan MacGregor for her help in reviewing and editing the field-test report. This study was supported by the United States President's Emergency Plan for AIDS Relief (PEPFAR) through the U.S. Centers for Disease Control and Prevention (CDC), Atlanta. The Associate Director for Science at the Center for Global Health of the CDC approved this study, and permission to conduct the field test was granted by the Kenya Ministry of Health (MOH).

\section{References}

1. Hermans SM, van Leth F, Manabe YC, Hoepelman AI, Lange JM, et al. 2012. Earlier initiation of antiretroviral therapy, increased tuberculosis case finding and reduced mortality in a setting of improved HIV care: a retrospective cohort study. HIV Med. 13(6), 337-44. doi:http://dx.doi.org/10.1111/j.1468-1293.2011.00980.x. PubMed

2. Ford N, Kranzer K, Hilderbrand K, Jouquet G, Goemaere E, et al. 2010. Early initiation of antiretroviral therapy and associated reduction in mortality, morbidity and defaulting in a nursemanaged, community cohort in Lesotho. AIDS. 24(17), 2645-50. doi:http://dx.doi.org/10.1097/QAD.0b013e32833ec5b2. PubMed

3. WHO UNICEF and UNAIDS. Global HIVIAIDS response: epidemic update and health sector progress towards universal access: progress report 2011. 2012, World health Organization: Geneva.[Accessed May 07, 2013], Available from: http://www.who.int/hiv/pub/progress_report2011/en/index.html.

4. Schouten EJ, Jahn A, Midiani D, Makombe SD, Mnthambala A, et al. 2011. Prevention of motherto-child transmission of HIV and the health-related Millennium Development Goals: time for a public health approach. Lancet. 378(9787), 282-84. doi:http://dx.doi.org/10.1016/S0140$\underline{6736(10) 62303-3}$. PubMed

5. El-Sadr WM, Holmes CB, Mugyenyi P, Thirumurthy H, Ellerbrock T, et al. 2012. Scale-up of HIV treatment through PEPFAR: a historic public health achievement. J Acquir Immune Defic Syndr. 60(Suppl 3), S96-104. doi:http://dx.doi.org/10.1097/QAI.0b013e31825eb27b. PubMed

6. Williams F, Boren SA. 2008. The role of the electronic medical record (EMR) in care delivery development in developing countries: a systematic review. Inform Prim Care. 16(2), 139-45. PubMed 
7. UNAIDS. 90-90-90 An ambitious treatment target to help end the AIDS epidemic. 2014 [Accessed 2016 March]; Available from: http://www.unaids.org/sites/default/files/media_asset/90-9090_en_0.pdf.

8. The Office of the Global AIDS Coordinator. PEPFAR Blueprint: Creating an AIDS-free Generation. 2012 [Accessed 2013 June 19]; Available from: http://www.pepfar.gov/documents/organization/201386.pdf.

9. The Office of the U.S. Global AIDS Coordinator. PEPFAR 3.0. Controlling the epidemic: delivering on the promise of an AIDS-free generation., PEPFAR, Editor. 2014: Washington (DC)Available from: http://www.pepfar.gov/documents/organization/234744.pdf

10. Ministry of Health Kenya. National e-Health Strategy 2011-2017. 2011.[Accessed March 27, 2014], Available from: http://www.isfteh.org/files/media/kenya_national_ehealth_strategy_20112017.pdf

11. WHO-ITU. National eHealth Strategy Toolkit. 2012, World Health Organization and International Telecommunication Union Geneva, SwitzerlandAvailable from: https://www.itu.int/dms_pub/itud/opb/str/D-STR-E_HEALTH.05-2012-PDF-E.pdf.

12. Oluoch T, Santas X, Kwaro D, Were M, Biondich P, et al. 2012. The effect of electronic medical record-based clinical decision support on HIV care in resource-constrained settings: a systematic review. Int J Med Inform. 81(10), e83-92. doi:http://dx.doi.org/10.1016/j.ijmedinf.2012.07.010. $\underline{\text { PubMed }}$

13. Porter LE, Bouey PD, Curtis S, Hochgesang M, Idele P, et al. 2012. Beyond indicators: advances in global HIV monitoring and evaluation during the PEPFAR era. J Acquir Immune Defic Syndr. 60(Suppl 3), S120-26. doi:http://dx.doi.org/10.1097/QAI.0b013e31825cf345. PubMed

14. Chan M, Kazatchkine M, Lob-Levyt J, Obaid T, Schweizer J, et al. 2010. Meeting the demand for results and accountability: a call for action on health data from eight global health agencies. PLoS Med. 7(1), e1000223. doi:http://dx.doi.org/10.1371/journal.pmed.1000223. PubMed

15. Manya ABJ, Øverland L, Titlestand O, Mumo J, Nzioka C. National Roll out of District Health Information Software (DHIS 2) in Kenya, 2011 - Central Server and Cloud based Infrastructure. in IST-Africa 2012 Conference Proceedings. 2012. Dar es Salaam, Tanzania.

16. Kihuba E, Gathara D, Mwinga S, Mulaku M, Kosgei R, et al. 2014. Assessing the ability of health information systems in hospitals to support evidence-informed decisions in Kenya. Glob Health Action. 7, 24859. doi:http://dx.doi.org/10.3402/gha.v7.24859. PubMed

17. Smyth ETMG, Barr JG, Dickson LM, Thompson IM. 1997. Automated entry of hospital infection surveillance data. Infect Control Hosp Epidemiol. 18(7), 486-91. $\underline{\text { PubMed }}$ http://dx.doi.org/10.2307/30141188

18. Thompson IM. 1999. Automated entry of nosocomial infection surveillance data: use of an optical scanning system. J Hosp Infect. 43(Suppl), S275-78. PubMed http://dx.doi.org/10.1016/S01956701(99)90099-3 
19. Fu PC, Jr, Rosenthal D, Pevnick JM, Eisenberg F. 2012. The impact of emerging standards adoption on automated quality reporting. $J$ Biomed Inform. 45(4), 772-81. doi:http://dx.doi.org/10.1016/j.jbi.2012.06.002. PubMed

20. Mamlin BW, Biondich PG, Wolfe BA, Fraser H, Jazayeri D, et al. 2006. Cooking up an open source EMR for developing countries: OpenMRS - a recipe for successful collaboration. AMIA Annu Symp Proc. 1, 529-33. PubMed

21. Open Health News. District Health Information System 2 (DHIS2). 2014 [Accessed 2016 April 13]; Available from: http://www.openhealthnews.com/resources/district-health-informationsystem-2-dhis2.

22. Kariuki J, Manders E, Richards J, Oluoch T, Mulonzi J and Kimanga D, Automating indicator data reporting from an EMR to aggregate data system using OpenMRS and DHIS2. Journal of Health Informatics in Africa, 2013. 1(1): p. 7http://dx.doi.org/10.12856/JHIA-2013-v1-i1.

23. International Association for Information and Data Quality. IQ/DQ glossary. 2015 July 19, 2015 [Accessed 2016 April 04,]; Available from: http://iaidq.org/main/glossary.shtml.

24. National AIDS and STI Control programme, MOH Comprehensive HIV Care and Treatment Facility Reporting form. NASCOP: Nairobi.[Accessed October 15, 2013], Available from: http://nascop.or.ke/library/3d/MOH_731.pdf.

25. Kariuki J, Manders E, Richards J. Interoperability Guide for Indicator Data Reporting: Automation of indicator data reporting from OpenMRS to DHIS 2. 2014; Available from: http://confluence.phiresearchlab.org/confluence/display/LAB/OpenMRS+to+DHIS+2+indicator + data+reporting.

26. National AIDS and STI Control programme, NASCOP indicator manual. 2009, NASCOP: Nairobi.[Accessed October 15, 2013], Available http://nascop.or.ke/library/3d/NASCOP\%20Harmonized\%20\%20Indicator\%20Manual.pdf.

27. Tonnesen AS, LeMaistre A, Tucker D. 1999. Electronic medical record implementation barriers encountered during implementation. Proc AMIA Symp. •••, 624-26. PubMed

28. Waters E, Rafter J, Douglas GP, Bwanali M, Jazayeri D, et al. 2010. Experience implementing a point-of-care electronic medical record system for primary care in Malawi. Stud Health Technol Inform. 160(Pt 1), 96-100. PubMed

29. Douglas GP, Gadabu OJ, Joukes S, Mumba S, McKay MV, et al. 2010. Using touchscreen electronic medical record systems to support and monitor national scale-up of antiretroviral therapy in Malawi. PLoS Med. 7(8). doi:http://dx.doi.org/10.1371/journal.pmed.1000319. PubMed

30. Mphatswe W, Mate KS, Bennett B, Ngidi H, Reddy J, et al. 2012. Improving public health information: a data quality intervention in KwaZulu-Natal, South Africa. Bull World Health Organ. 90(3), 176-82. doi:http://dx.doi.org/10.2471/BLT.11.092759. PubMed 
31. Ledikwe JH, Grignon J, Lebelonyane R, Ludick S, Matshediso E, et al. 2014. Improving the quality of health information: a qualitative assessment of data management and reporting systems in Botswana. Health Res Policy Syst. 12, 7. doi:http://dx.doi.org/10.1186/1478-4505-12-7. PubMed 


\section{Appendices}

Appendix 1: MOH 731 HIV/AIDS Facility Reporting Form

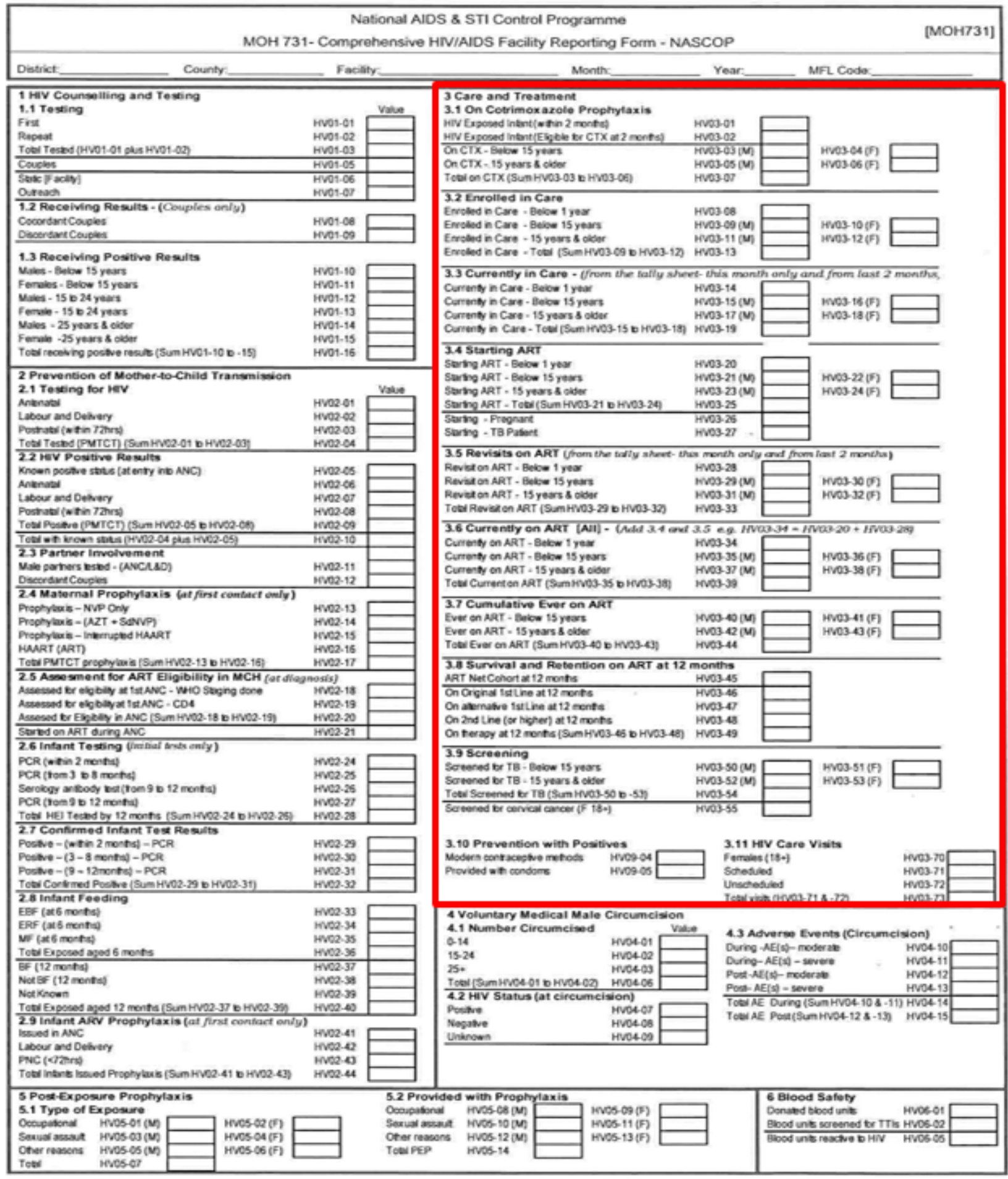

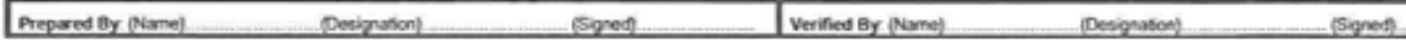

NOTE: Care and Treatment indicators enclosed in the red box 